\title{
Identification of microRNAs involved in growth arrest and cell death in hydrogen peroxide-treated human dermal papilla cells
}

\author{
OK-YEON KIM ${ }^{1 *}$, HWA JUN CHA ${ }^{1,2^{*}}$, KYU JOONG AHN ${ }^{3}$, IN-SOOK AN ${ }^{2,4}$, \\ SUNGKWAN AN ${ }^{1,2,4}$ and SEUNGHEE BAE ${ }^{1,2}$ \\ ${ }^{1}$ Molecular-Targeted Drug Research Center, ${ }^{2}$ Korea Institute for Skin and Clinical Sciences, Konkuk University; \\ ${ }^{3}$ Department of Dermatology, Konkuk University School of Medicine; ${ }^{4}$ Department of Cosmetology, \\ Graduate School of Engineering, Konkuk University, Seoul 143-701, Republic of Korea
}

Received July 31, 2013; Accepted March 12, 2014

DOI: $10.3892 / \mathrm{mmr} .2014 .2158$

\begin{abstract}
RNAs (miRNAs) are small non-coding RNAs that regulate various biological processes by interfering with the translation of target genes. Several studies have suggested that miRNAs are involved in cellular responses to hydrogen peroxide $\left(\mathrm{H}_{2} \mathrm{O}_{2}\right)$. Reactive oxygen species (ROS) are involved in hair malignancies, however, the $\mathrm{H}_{2} \mathrm{O}_{2}$-induced, miRNA-dependent regulatory mechanisms of human dermal papilla (HDP) cells are not fully understood. Our previous study demonstrated that changes in miRNA expression function to regulate growth arrest and apoptosis in UVB-irradiated HDPs. In the present study, miRNA expression was profiled in HDPs treated with $\mathrm{H}_{2} \mathrm{O}_{2}$. The transcriptome analysis of $\mathrm{H}_{2} \mathrm{O}_{2}$-treated HDPs enabled the identification of 68 differentially expressed miRNAs (62 were upregulated and 6 were downregulated) and 14,316 putative target genes of the miRNAs. Gene ontology (GO) analysis was utilized to verify that the putative target genes of the altered miRNAs were associated with $\mathrm{H}_{2} \mathrm{O}_{2}$-induced cell growth arrest and apoptosis. This bioinformatics analysis indicated that $\mathrm{H}_{2} \mathrm{O}_{2}$-response pathways involved in growth arrest and apoptosis were significantly affected. The identification of miRNAs and their putative targets may offer new therapeutic strategies for $\mathrm{H}_{2} \mathrm{O}_{2}$-induced hair follicle disorders.
\end{abstract}

Correspondence to: Dr Seunghee Bae, Molecular-Targeted Drug Research Center and Korea Institute for Skin and Clinical Sciences, Konkuk University, 120 Neungdong-ro, Gwangjin-gu, Seoul 143-701, Republic of Korea

E-mail: sbae@konkuk.ac.kr

*Contributed equally

Key words: microRNA, hydrogen peroxide, human dermal papilla cells, microarray

\section{Introduction}

The dermal papilla (DP) describes the component of the hair follicle that is involved in hair growth and formation. During development of the hair follicle, the DP is generated by condensation of dermal mesenchymal cells. The DP exists at the base of hair follicles and regulates the hair cycle by providing key signals that control the timing and phase of hair follicle growth and formation $(1,2)$. Anagen is the active growth phase of hair follicles, where the root divides rapidly and adds material to the hair follicle for rapid hair growth. DP stimulates the initiation of the anagen growth phase by secreting Fgf7/10 and TGF $\beta(3,4)$. DP-induced $\beta$-catenin/Wnt signaling sustains the anagen phase and DP-induced Notch/Wnt5a signaling induces hair follicle differentiation $(5,6)$. The production of reactive oxygen species (ROS) in hair follicles results in hair developmental disorders, including graying and hair loss (7-9). One such example is alopecia, which is induced by a ROS-mediated reduction of hair growth $(9,10)$.

Oxidative stress that results in crucial damage of DNA, proteins and lipids is implicated in several hair follicle disorders, including graying and hair loss (7-10). Oxidative stress is increased by the accumulation of ROS, including hydrogen peroxide $\left(\mathrm{H}_{2} \mathrm{O}_{2}\right)$, hydroxyl radical $\left(\mathrm{OH}^{*}\right)$, superoxide anion $\left(\mathrm{O}^{-}\right)$and the accumulation of reactive nitrogen species (RNS), including peroxynitrite (ONOO-). The generation of ROS and RNS is triggered by an imbalance between pro-oxidants and antioxidants. It has been identified that high levels of oxidative stress induces growth arrest, apoptosis and necrosis in cells (11). Mammalian cells have developed defense mechanisms to neutralize ROS, including antioxidant enzymes and non-enzymatic antioxidants $(12,13)$. In hair follicles, low levels of ROS are generated in the mitochondria, and these act as crucial signaling molecules for hair follicle differentiation and morphogenesis (7). However, high levels of ROS directly damages cellular membranes, lipids, proteins and DNA (9). ROS levels increase with aging, which causes a decrease in the function and number of functional melanocyte cells in hair follicles (8). ROS-responsive microRNA (miRNA) 
expression regulates the cell cycle and apoptosis in a variety of cells (14-16).

miRNAs are short oligonucleotides, consisting of $\sim 19-24$ nucleotides (17). miRNAs repress the translation of their target genes by binding to partly complementary sequences in the 3 ' untranslated region of the target mRNA (18). miRNAs are involved in the control of diverse cellular processes, including cell growth, apoptosis, development, metabolism, stress adaptation, hormone signaling and differentiation (18-22). In hair follicles, a deficiency of miRNAs induced by the knockout of Dicer and Drosha blocks the anagen developmental phase by repression of the catagen phase (23). It has been hypothesized that the miRNA miR-31 is involved in hair follicle growth and hair fiber formation because it targets $\mathrm{Krt16}, \mathrm{Krt17}, \mathrm{Dl} \times 3$ and FgflO (24). A recent study compared the miRNA expression profiles in balding and non-balding dermal papilla (25).

To the best of our knowledge, no previous studies have reported the miRNA expression profile in $\mathrm{H}_{2} \mathrm{O}_{2}$-treated DP. Therefore, in the present study, we analyzed changes in the miRNA expression profiles in DP alone and in DP that had been treated with $\mathrm{H}_{2} \mathrm{O}_{2}$. The target genes of significant miRNAs (those with $>2$-fold changes in expression) were predicted by an in silico prediction algorithm. Based on these data, we derive a model indicating that $\mathrm{H}_{2} \mathrm{O}_{2}$-specific miRNAs regulate ROS-responsive cellular functions.

\section{Materials and methods}

Cell culture. Human dermal papilla (HDP) cells were purchased from Cellbio Inc. (Seoul, Korea). HDPs were maintained as a monolayer culture in Dulbecco's modified Eagle's medium (DMEM; Gibco-Invitrogen Life Technologies, Carlsbad, CA, USA) supplemented with $10 \%$ fetal bovine serum (FBS; Sigma-Aldrich, St. Louis, MO, USA) and 1\% penicillin/streptomycin. HDPs were cultured in a humidified chamber with $5 \% \mathrm{CO}_{2}$ at $37^{\circ} \mathrm{C}$.

Cell viability. HDPs ( $5 \times 10^{3}$ cells) were plated in 96-well culture plates and treated with $\mathrm{H}_{2} \mathrm{O}_{2}$ for $24 \mathrm{~h}$ under the growth conditions described above. The 3-(4,5-dimethylthiazol-2-yl)-2,5-diphenyltetrazolium bromide (MTT) colorimetric assay was performed by adding $0.5 \mu \mathrm{g} / \mathrm{ml}$ MTT (Sigma-Aldrich) to the culture medium. Following $1 \mathrm{~h}$ of incubation under normal cell growth conditions, the growth medium was removed and $200 \mu 1$ of dimethyl sulfoxide was added to each well. The absorbance was measured at $490 \mathrm{~nm}$ using a microplate reader (iMark; Bio-Rad, Hercules, CA, USA).

Cell cycle analysis. The cell cycle was determined by flow cytometry via propidium iodide (PI) staining. Cell cycle analysis was performed as described previously (26).

RNA preparation and miRNA microarray. Total RNAs were extracted by RiboEX (GeneAll Biotechnology Co., Ltd., Seoul, Korea) and quantified by measuring the optical density ratio. The miRNA microarray (SurePrint G3 Human v16.0 miRNA 8x60K; Agilent Technologies, Santa Clara, CA, USA) was performed according to the manufacturer's instructions. miRNA was stained by pCp-Cy3 (Agilent Technologies), combined with T4 ligase (Agilent Technologies) and hybrid- ized to a probe on the microarray. The microarray was imaged using the Agilent microarray scanner and digitized by Feature extraction. The digitized data were analyzed for fold change, miRNA potential target and gene ontology (GO) using Genespring GX version 11.5 (Agilent Technologies).

Prediction of miRNA target genes and GO analysis. The putative target genes of significantly up and downregulated miRNAs were identified by the web tool TargetScan. Target gene prediction was performed on significant miRNAs with 50 context score percentile using the conserved and non-conserved database. The putative target genes were identified and sorted by GO of each gene.

Statistical analysis. Statistical significance was determined by Student's t-test. $\mathrm{P}<0.05$ was considered to indicate a statistically significant difference.

\section{Results}

$\mathrm{H}_{2} \mathrm{O}_{2}$ treatment decreases the viability of $\mathrm{HDPs}$ via cell cycle arrest or apoptosis. To determine the cytotoxicity of $\mathrm{H}_{2} \mathrm{O}_{2}$, we initially examined the viability and status of the $\mathrm{G} 1$ or $\mathrm{G} 2 / \mathrm{M}$ population in HDPs. HDPs were treated with $\mathrm{H}_{2} \mathrm{O}_{2}$ $(0-1,000 \mu \mathrm{M})$ and the resulting viability was measured using the MTT assay (Fig. 1A). Following 24 h of $\mathrm{H}_{2} \mathrm{O}_{2}$ treatment, a decrease in cell viability occurred in a dose-dependent manner. In the presence of 750 and $1,000 \mu \mathrm{M} \mathrm{H}_{2} \mathrm{O}_{2}$, a significant $(\mathrm{P}<0.05)$ decrease in cell viability to 71.23 and $50.60 \%$ of that in control cells, respectively, was observed (Fig. 1A). In the presence of $750 \mu \mathrm{M} \mathrm{H}_{2} \mathrm{O}_{2}$, HDPs exhibited a $21.07 \%$ reduction in $\mathrm{G} 1$ phase and an $11.66 \%$ increase in $\mathrm{G} 2 / \mathrm{M}$ phase, compared with those of the control (Fig. 1B). These data suggest that $\mathrm{H}_{2} \mathrm{O}_{2}$ decreases cell viability of HDPs by inducing cell cycle arrest at the $\mathrm{G} 2 / \mathrm{M}$ phase and cell death.

Identification of $\mathrm{H}_{2} \mathrm{O}_{2}$-induced changes in miRNA expression in HDPs. $\mathrm{H}_{2} \mathrm{O}_{2}$-induced changes in the miRNA expression profiles were analyzed using the Agilent miRNA microarray, which contains 1,368 probes that are able to detect 1,205 human miRNAs. The fluorescence intensity data for each sample was normalized by global normalization. To eliminate disorderly data, miRNA expression data were selected by requiring a present-flag for at least one of all samples. Using this method, 155 miRNAs were selected out of 1,205 human miRNAs detected by present-flag selection. To identify $\mathrm{H}_{2} \mathrm{O}_{2}$-regulated miRNAs, the refined data were compared for control HDPs grown under normal conditions and HDPs treated with $750 \mu \mathrm{M}$ $\mathrm{H}_{2} \mathrm{O}_{2}$ for $24 \mathrm{~h}$. The results demonstrated that the expression levels of 68 miRNAs were altered at least 1.5 -fold in response to treatment with $\mathrm{H}_{2} \mathrm{O}_{2}$. The 68 miRNAs are presented in Fig. 2 . Fold-change analysis revealed that 62 miRNAs are upregulated and 6 miRNAs are downregulated at levels of 1.5-fold or greater in control HDPs grown under normal conditions and HDPs treated with $750 \mu \mathrm{M} \mathrm{H}_{2} \mathrm{O}_{2}$ for $24 \mathrm{~h}$ (Table I).

Identification of $\mathrm{H}_{2} \mathrm{O}_{2}$-specific miRNA putative target genes and GO analysis. Our study identified 68 novel miRNAs that were significantly up or downregulated in response to $\mathrm{H}_{2} \mathrm{O}_{2}$ treatment. As miRNA functions as RNA interference during mRNA 
A

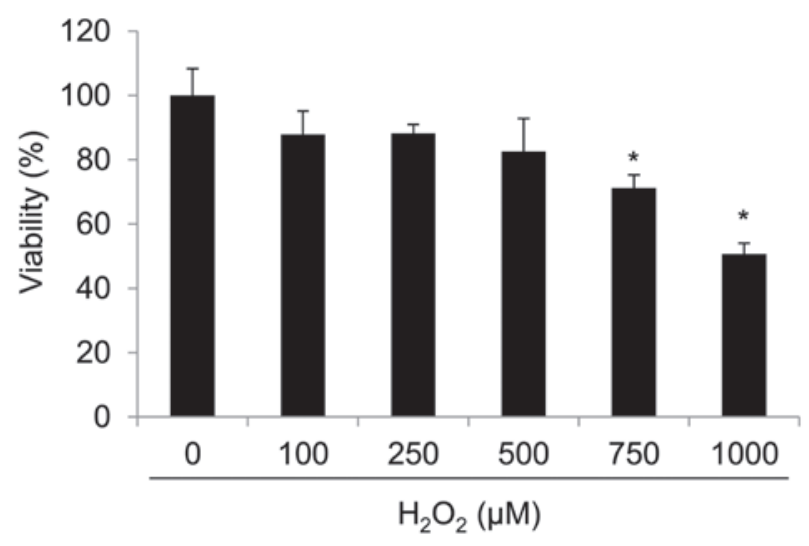

B

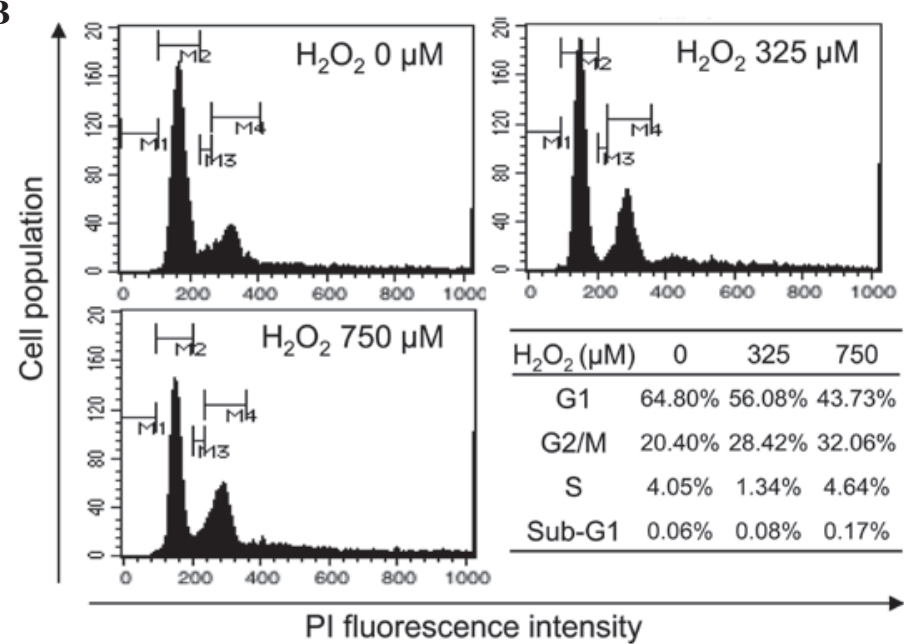

Figure $1 . \mathrm{H}_{2} \mathrm{O}_{2}$ repression of cell growth via cell cycle arrest and apoptosis in HDP cells. (A) Growth properties of $\mathrm{H}_{2} \mathrm{O}_{2}$-treated $\mathrm{HDPs}$. $\mathrm{HDPs}\left(5 \times 10^{3}\right)$ were seeded in 96-well plates and treated with the indicated $\mathrm{H}_{2} \mathrm{O}_{2}$ concentrations, incubated for $24 \mathrm{~h}$ and growth properties were measured using the MTT assay. Cell viability (average $\pm \mathrm{SD}$ ) was determined from triplicate experiments ( $\mathrm{P}<0.05)$. (B) HDPs $\left(2 \times 10^{6}\right)$ were seeded in a 60 -mm culture dish and treated with $0-750 \mu \mathrm{M} \mathrm{H}_{2} \mathrm{O}_{2}$, incubated for $24 \mathrm{~h}$ and processed for flow cytometry by PI staining. Graphical representation of FACS data, bar graph represents percentage of total cell population in each cell cycle phase $( \pm \mathrm{SD})$. $\mathrm{H}_{2} \mathrm{O}_{2}$, hydrogen peroxide; HDP, human dermal papilla; PI, propidium iodide.

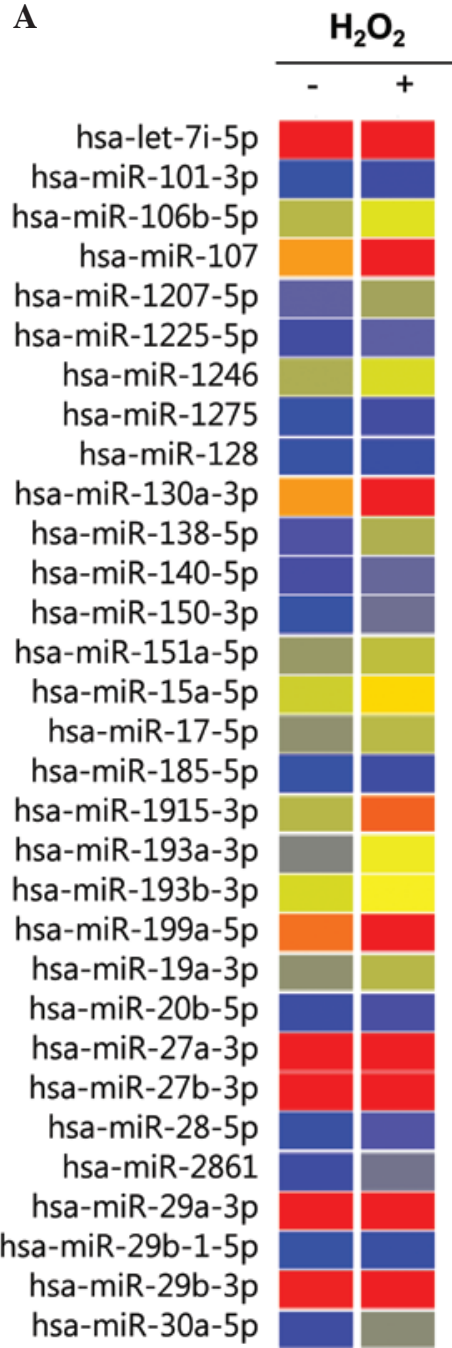

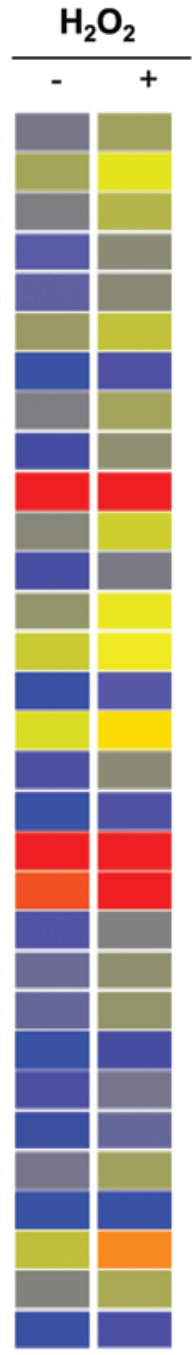

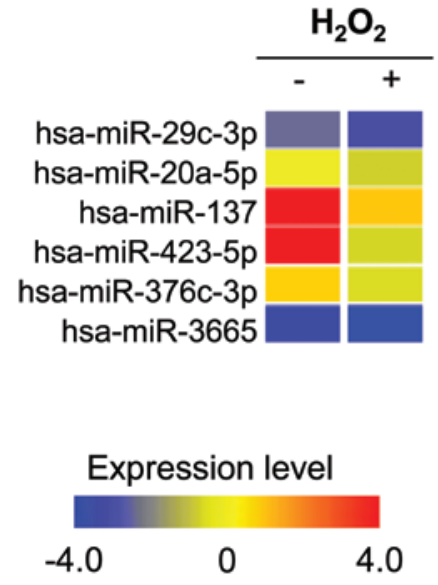

B

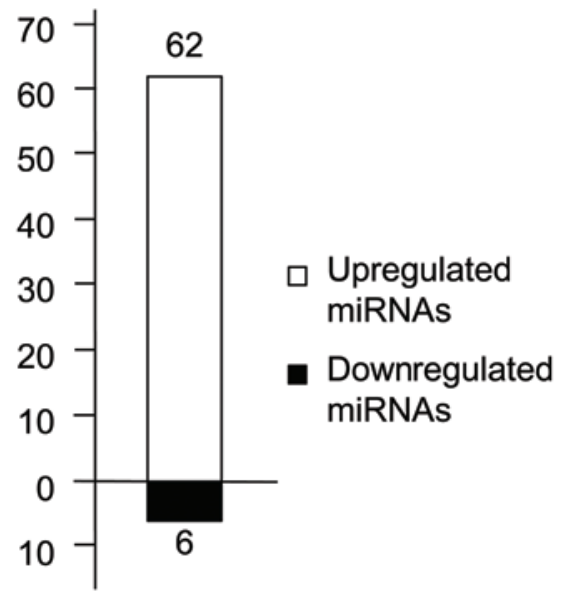

Figure 2. Changes in miRNA expression levels in $\mathrm{H}_{2} \mathrm{O}_{2}$-treated HDP cells. (A) Total RNA was extracted from control HDPs and HDPs treated with $750 \mu \mathrm{M}$ $\mathrm{H}_{2} \mathrm{O}_{2}$ for $24 \mathrm{~h}$. miRNA microarray was performed as described in Materials and methods. To obtain the fold change in expression levels, the fluorescence intensity of each miRNA was compared between normal HDPs and $\mathrm{H}_{2} \mathrm{O}_{2}$-treated HDPs. Changes in miRNA expression $>2$-fold are illustrated by heat map. Blue indicates a relatively low expression and red indicates a relatively high expression. (B) Up and downregulated miRNAs in response to $\mathrm{H}_{2} \mathrm{O}_{2}$ treatment were counted and represented by the bar graph. miRNA, microRNA; $\mathrm{H}_{2} \mathrm{O}_{2}$, hydrogen peroxide; HDP, human dermal papilla. 
Table I. Up and downregulated miRNAs in $\mathrm{H}_{2} \mathrm{O}_{2}$-treated HDPs.

A, Upregulated

\begin{tabular}{|c|c|c|c|c|c|c|c|c|}
\hline miRNA & Fold change & Chr & miRNA & Fold change & Chr & miRNA & Fold change & Chr \\
\hline hsa-let-7i-5p & 1.52 & $\operatorname{chr} 12$ & hsa-miR-19a-3p & 1.52 & $\operatorname{chr} 13$ & hsa-miR-361-5p & 1.86 & $\operatorname{chrX}$ \\
\hline hsa-miR-101-3p & 1.91 & chr1 & hsa-miR-20b-5p & 1.56 & $\operatorname{chrX}$ & hsa-miR-3663-3p & 2.73 & $\operatorname{chr} 10$ \\
\hline hsa-miR-106b-5p & 1.59 & chr7 & hsa-miR-27a-3p & 2.13 & chr19 & hsa-miR-376a-3p & 1.65 & chr14 \\
\hline hsa-miR-107 & 1.55 & $\operatorname{chr} 10$ & hsa-miR-27b-3p & 1.56 & $\operatorname{chr} 9$ & hsa-miR-376b-3p & 2.39 & $\operatorname{chr} 14$ \\
\hline hsa-miR-1207-5p & 2.08 & chr8 & hsa-miR-28-5p & 2.17 & $\operatorname{chr} 3$ & hsa-miR-4281 & 2.15 & $\operatorname{chr} 5$ \\
\hline hsa-miR-1225-5p & 1.62 & $\operatorname{chr} 16$ & hsa-miR-2861 & 2.29 & $\operatorname{chr} 9$ & hsa-miR-4306 & 1.65 & $\operatorname{chr} 13$ \\
\hline hsa-miR-1246 & 1.60 & $\operatorname{chr} 2$ & hsa-miR-29a-3p & 1.60 & $\operatorname{chr} 7$ & hsa-miR-487b & 1.51 & $\operatorname{chr} 14$ \\
\hline hsa-miR-1275 & 1.75 & chr6 & hsa-miR-29b-1-5p & 2.14 & $\operatorname{chr} 7$ & hsa-miR-503-5p & 1.66 & $\operatorname{chrX}$ \\
\hline hsa-miR-128 & 1.54 & $\operatorname{chr} 2$ & hsa-miR-29b-3p & 3.26 & chr1 & hsa-miR-574-3p & 2.01 & chr4 \\
\hline hsa-miR-130a-3p & 1.63 & $\operatorname{chr} 11$ & hsa-miR-30a-5p & 3.01 & chr6 & hsa-miR-630 & 1.66 & $\operatorname{chr} 15$ \\
\hline hsa-miR-138-5p & 2.84 & chr3 & hsa-miR-31-3p & 1.58 & chr9 & hsa-miR-638 & 2.33 & $\operatorname{chr} 19$ \\
\hline hsa-miR-140-5p & 1.55 & $\operatorname{chr} 16$ & hsa-miR-3162-5p & 2.00 & chr11 & hsa-miR-654-3p & 1.63 & $\operatorname{chr} 14$ \\
\hline hsa-miR-150-3p & 3.95 & $\operatorname{chr} 19$ & hsa-miR-3195 & 1.81 & $\operatorname{chr} 20$ & hsa-miR-758-3p & 3.00 & $\operatorname{chr} 14$ \\
\hline hsa-miR-151a-5p & 1.52 & chr8 & hsa-miR-3196 & 1.67 & $\operatorname{chr} 20$ & hsa-miR-762 & 2.34 & $\operatorname{chr} 16$ \\
\hline hsa-miR-15a-5p & 1.76 & $\operatorname{chr} 13$ & hsa-miR-320c & 1.52 & $\operatorname{chr} 18$ & hsa-miR-92a-3p & 1.51 & $\operatorname{chr} 13$ \\
\hline hsa-miR-17-5p & 1.56 & $\operatorname{chr} 13$ & hsa-miR-320d & 1.52 & $\operatorname{chr} 13$ & hsa-miR-99b-5p & 2.01 & $\operatorname{chr} 19$ \\
\hline hsa-miR-185-5p & 1.64 & $\operatorname{chr} 22$ & hsa-miR-324-3p & 2.38 & $\operatorname{chr} 17$ & hsa-miR-377-3p & 1.55 & $\operatorname{chr} 14$ \\
\hline hsa-miR-1915-3p & 2.81 & $\operatorname{chr} 10$ & hsa-miR-331-3p & 1.51 & $\operatorname{chr} 12$ & hsa-miR-381-3p & 2.03 & $\operatorname{chr} 14$ \\
\hline hsa-miR-193a-3p & 3.65 & $\operatorname{chr} 17$ & hsa-miR-337-5p & 2.52 & $\operatorname{chr} 14$ & hsa-miR-411-5p & 2.47 & $\operatorname{chr} 14$ \\
\hline hsa-miR-193b-3p & 1.56 & chr16 & hsa-miR-34a-5p & 2.04 & chr1 & hsa-miR-424-5p & 1.72 & $\operatorname{chrX}$ \\
\hline hsa-miR-199a-5p & 1.61 & chr1 & hsa-miR-34b-5p & 2.19 & chr11 & & & \\
\hline
\end{tabular}

B, Downregulated

\begin{tabular}{lcccccccc}
\hline miRNA & Fold change & Chr & miRNA & Fold change & Chr & miRNA & Fold change & Chr \\
\hline hsa-miR-29c-3p & -1.51 & chr1 & hsa-miR-137 & -1.50 & chr1 & hsa-miR-376c-3p & -1.56 & chr14 \\
hsa-miR-20a-5p & -1.51 & chr13 & hsa-miR-423-5p & -1.57 & chr17 & hsa-miR-3665 & -2.36 & chr13 \\
\hline
\end{tabular}

miRNAs, microRNAs; $\mathrm{H}_{2} \mathrm{O}_{2}$, hydrogen peroxide; HDPs, human dermal papilla cells.

translation, we predicted that $\mathrm{H}_{2} \mathrm{O}_{2}$-specific miRNAs may regulate all or a number of the $\mathrm{H}_{2} \mathrm{O}_{2}$-response genes. Therefore, the target genes of $\mathrm{H}_{2} \mathrm{O}_{2}$-specific miRNAs were analyzed using the bioinformatics target gene prediction program TargetScan. For target gene analysis, default parameters were utilized, 50 context score percentile in the conserved and nonconserved database. $\mathrm{H}_{2} \mathrm{O}_{2}$-induced miRNAs targeted 14,046 genes; $\mathrm{H}_{2} \mathrm{O}_{2}$-repressed miRNAs targeted 6,019 genes. To identify the cellular functions of the putative target genes, GO analysis was performed, which is a method that categorizes the genes according to the cellular function classified for a standard for each gene. As illustrated in Fig. 3, GO analysis identified the following cellular functional activities: for molecular function, catalytic (17.01\%), nucleic acid binding transcription factor (6.67\%), enzyme regulator $(2.62 \%)$, molecular transducer (2.61\%), binding $(67.63 \%)$ and transporter (3.45\%); for biological process, signaling (7.29\%), biological adhesion (1.65\%), multicellular organismal (4.11\%), cellular $(18.52 \%)$, metabolic $(13.96 \%)$, cellular component organization or biogenesis $(0.63 \%)$, immune system $(0.01 \%)$, biological regulation (14.89\%), establishment of localization (6.16\%), localization
(6.21\%), response to stimulus (7.15\%), single organism (15.35\%) and developmental (4.06\%); for cellular component part, cell $(25.45 \%)$, membrane $(10.69 \%)$, organelle $(1.93 \%)$, extracellular region $(0.75 \%)$, extracellular matrix $(0.2 \%)$, organelle $(15.01 \%)$, membrane-enclosed lumen $(1.05 \%)$, cell junction $(0.96 \%)$, extracellular matrix $(0.77 \%)$, membrane $(14.3 \%)$, cell $(25.45 \%)$, extracellular region $(2.82 \%)$ and synapse $(0.61 \%)$. All of these were implicated in UVB-mediated responses in HDPs. These GO annotations provided comprehensive information on the function of $\mathrm{H}_{2} \mathrm{O}_{2}$-regulated transcripts in HDPs. TargetScan was used to predict the gene targets of the top five miRNAs that demonstrated the greatest increase or decrease in expression levels. The putative miRNA target genes were sorted into cell cycle, apoptosis and cell growth, and proliferation-related GO (Tables II and III). Cell cycle-related GO included cell cycle (GO:0007049), cell cycle arrest (GO:0007049), negative regulation of cell cycle (GO:0045786) and regulation of cell cycle (GO:0051726). Apoptosis-related GO included apoptotic process (GO:0006915), apoptotic signaling pathway (GO:0097190), cell death (GO:0008219), death (GO:0016265), 


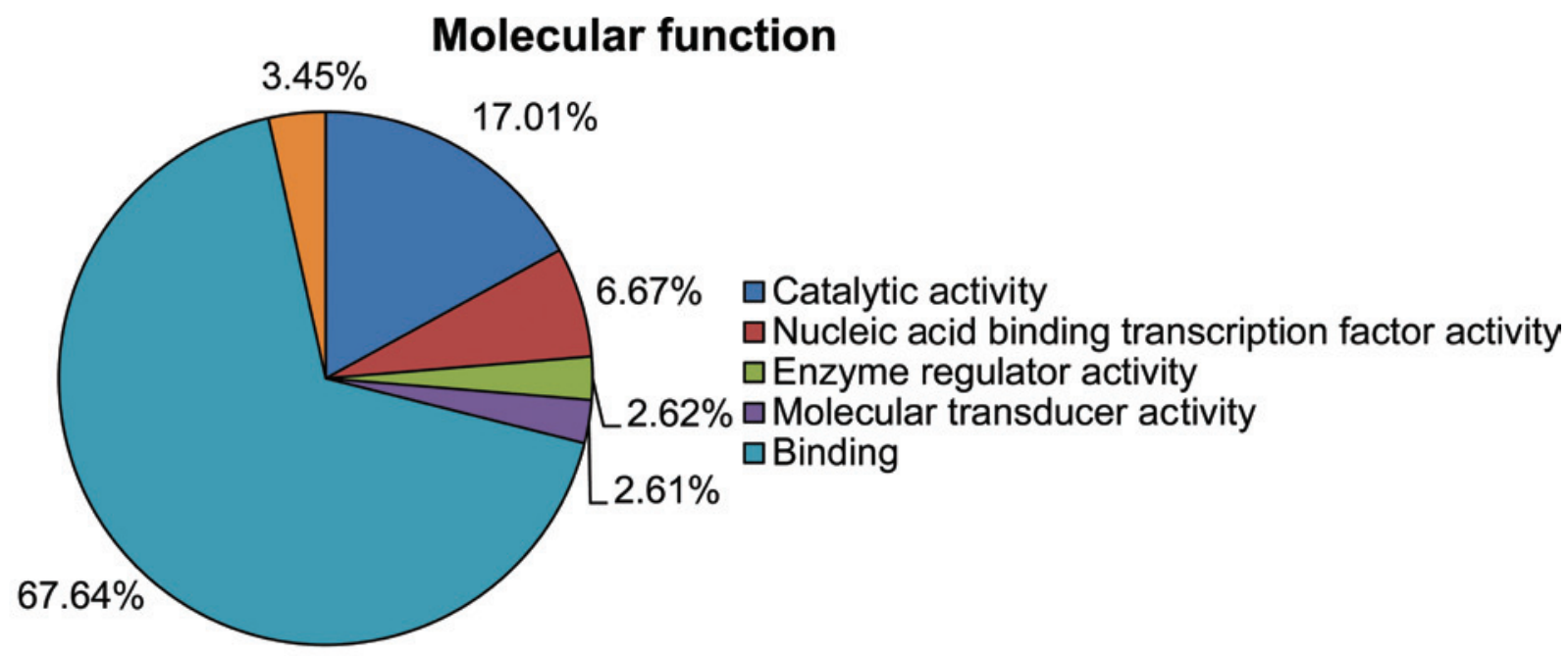

\section{Biological process}

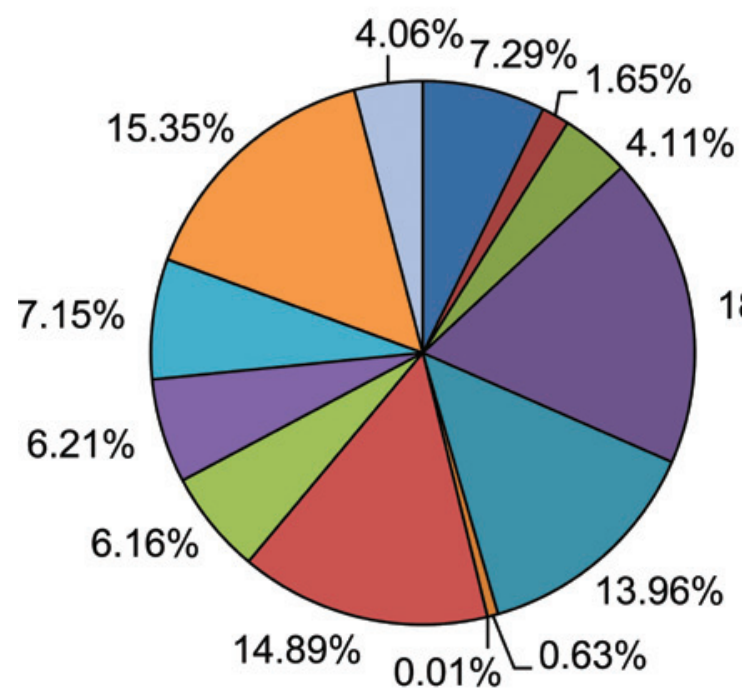

$\square$ Signaling

$\square$ Biological adhesion

口Multicellular organismal process

$\square$ Cellular process

$18.52 \%$

aMetabolic process

$\square$ Cellular component organization or biogenesis

口Immune system process

$\square$ Biological regulation

口Establishment of localization

-Localization

$\square$ Response to stimulus

$\square$ Single organism process

口Developmental process

\section{Cellular component}

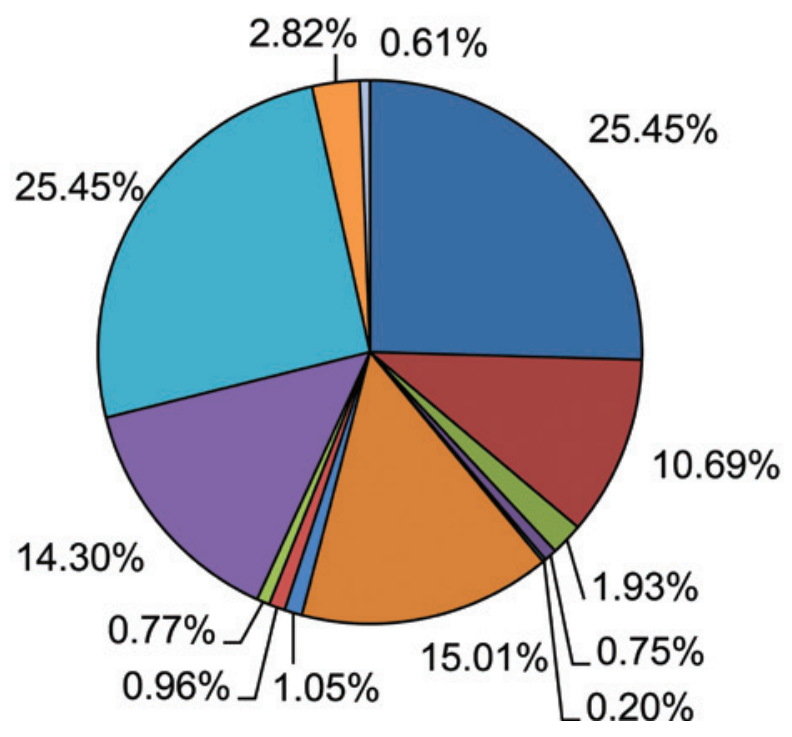

$\square$ Cell part

口Membrane part

口Organelle part

$\square$ Extracellular region part

$\square$ Extraceluular matrix part

口Organelle part

口Membrane-enclosed lumen

$\square$ Cell junction

口Extracellular matrix

aMembrane

$\square$ Cell

口Extacellular region

$\square$ Synapse

Figure 3. GO enrichment analysis of the target genes of up and downregulated miRNAs. Putative target genes of $\mathrm{H}_{2} \mathrm{O}_{2}$-specific miRNAs were identified by TargetScan and the cellular functions were predicted by GO analysis. The charts represent the GO analysis performed by Genespring GX. GO, gene ontology; miRNAs, microRNAs; $\mathrm{H}_{2} \mathrm{O}_{2}$, hydrogen peroxide. 
Table II. Predicted target genes of the top five most upregulated miRNAs in $\mathrm{H}_{2} \mathrm{O}_{2}$-treated HDPs.

Target genes and functions

\begin{tabular}{|c|c|c|c|}
\hline miRNA & Cell cycle & Apoptosis & Cell growth and proliferation \\
\hline hsa-miR-150-3p & - & - & - \\
\hline hsa-miR-193a-3p & $\begin{array}{l}\text { PSRC1, JUB, CCNT2, } \\
\text { CCPG1, DLGAP5, PAPD5, } \\
\text { SIAH1, TSC1, CABLES2, } \\
\text { SMC1A, ERBB2IP, SEP3, } \\
\text { KLK10, PTEN, CCND1, } \\
\text { NEDD9, NF1, NF2, SUFU, } \\
\text { CTF8, SASS6, E2F6, } \\
\text { SENP5, VASH1, } \\
\text { SPECC1L, CADM1, } \\
\text { APPL1, GAS1, ING1, } \\
\text { IRF1, FOXO4, ATM }\end{array}$ & $\begin{array}{l}\text { BCL2L10, DIDO1, } \\
\text { WDR92, RFFL, ZNF346, } \\
\text { CADM1, RNF144B, GAS1, } \\
\text { TNFRSF21, IL1A, JAK2, } \\
\text { MCL1, MLL, OSM, } \\
\text { SH3GLB1, PDCD2, } \\
\text { DRAM, PSEN1, BAG1, } \\
\text { BCL2L2, RTKN, ATXN1, } \\
\text { ELMO2, SIAH1, TIAL1, } \\
\text { TNFAIP3, TNFRSF1B, } \\
\text { TRAF1, PHLDA2, } \\
\text { DYRK2, AIFM2, TNFSF9, } \\
\text { TNFRSF10B, SQSTM1, } \\
\text { EBAG9, TP53INP1, } \\
\text { ARHGEF6, PPM1F }\end{array}$ & $\begin{array}{l}\text { BMPR2, TDGF1, } \\
\text { KAZALD1, PPP1R9B, } \\
\text { WISP1, EBAG9, SOCS6, } \\
\text { CIAO1, NET1, ENOX2, } \\
\text { TNFSF13B, FLT1, FLT3, } \\
\text { HOXD13, IGFBP5, KIT, } \\
\text { KRAS, NEDD9, NODAL, } \\
\text { OSM, CCDC88A, PRKCQ }\end{array}$ \\
\hline hsa-miR-29b-3p & $\begin{array}{l}\text { PPM1D, CDC23, CCND2, } \\
\text { CCNF, UBA3, CCNT2, } \\
\text { AURKB, HDAC4, } \\
\text { DCLRE1A, SUPT5H, } \\
\text { TACC1, PTP4A1, CDC7, } \\
\text { GSG2, SMPD3, RCC2, } \\
\text { PTEN, NEDD9, ZAK, } \\
\text { XRN1, CDK2, CDK6, } \\
\text { HTATIP2, CGREF1, } \\
\text { STAG2, SEP9, DBF4, } \\
\text { MAPRE2, E2F7, SLC5A8, } \\
\text { AHR, VASH1, MAPRE1, } \\
\text { CLASP2, SEP6 }\end{array}$ & $\begin{array}{l}\text { FEM1B, HTATIP2, IL24, } \\
\text { SLC5A8, AHR, FOXO3, } \\
\text { PPP1R13B, SIRT1, } \\
\text { ZNF346, CECR2, BIRC2, } \\
\text { FAS, IL2RA,, MCL1, } \\
\text { SH3GLB1, ZAK, CYCS, } \\
\text { DIABLO, DUSP22, } \\
\text { BIRC6, BAK1, ATXN1, } \\
\text { CIDEC, SGK1, ISG20L1, } \\
\text { TNFAIP3, TNFRSF1A, } \\
\text { TRAF5, FAM130A1, } \\
\text { CASP7, AIFM2, BMF, } \\
\text { RABEP1, TP53INP1, } \\
\text { TRAF4, SLK }\end{array}$ & $\begin{array}{l}\text { BIRC6, PURA, TRAF5, } \\
\text { VEGFA, EPC1, CDC7, } \\
\text { CREG1, WISP1, CCND2, } \\
\text { MORF4L2, CDK2, } \\
\text { MORF4L1, CHRNA7, } \\
\text { IFNG, IGF1, LIF, LIFR, } \\
\text { NDN, NEDD9, PDGFRB, } \\
\text { ING3, PPP2CA }\end{array}$ \\
\hline hsa-miR-30a-5p & $\begin{array}{l}\text { TBRG1, CCNK, CCNA1, } \\
\text { BCL10, LMLN, UBA3, } \\
\text { CCNT2, CCNE2, SEP7, } \\
\text { NEK4, TFDP1, TSC1, } \\
\text { UBE2I, PTP4A1, EVI5, } \\
\text { MIS12, SUV39H2, } \\
\text { CHAF1B, CDC7, RECK, } \\
\text { RGS2, RCBTB1, NEK1, } \\
\text { NF1, PNN, RBM5, STAG2, } \\
\text { DBF4, ESCO1, ESCO2, } \\
\text { DDX11, E2F3, EPHB2, } \\
\text { CLASP2, SEP6, CD2AP, } \\
\text { NSL1, MTBP, INCENP, } \\
\text { JAG2, KIF11, RHOB }\end{array}$ & $\begin{array}{l}\text { BCL2L11, EDAR, NLRP3, } \\
\text { RFFL, TICAM1, PRUNE2, } \\
\text { TRIM35, SIRT1, } \\
\text { RNF144B, TCTN3, GJA1, } \\
\text { CECR2, SH3KBP1, HTT, } \\
\text { HIP1, IL1A, IL2RA, } \\
\text { IL17A, MAP3K5, MLL, } \\
\text { MNT, BCL2L15, NAIP, } \\
\text { DDIT4,. C8orf4, AVEN, } \\
\text { PTGER3, BIRC6, TRIB3, } \\
\text { ATXN1, SOX9, ACTC1, } \\
\text { TFDP1, TIA1, CASP3, } \\
\text { UNC5C, TNFSF9, } \\
\text { TNFRSF10D, } \\
\text { TNFRSF10B, BCL10, } \\
\text { ATG12, EBAG9, DLG5, } \\
\text { ARHGEF6, ATG5 }\end{array}$ & $\begin{array}{l}\text { C19orf10, BIRC6, BNC1, } \\
\text { SOX9, VIPR1, CAMK2D, } \\
\text { CDC7, CDCA7, CUL3, } \\
\text { SOCS1, CREG1, OCS3, } \\
\text { EBAG9, CFDP1, ENOX2, } \\
\text { ADRA1D, ADRA2A, } \\
\text { ADRB2, DDX11, TLX1, } \\
\text { IL7, JAG2, KRAS, LIFR, } \\
\text { LYN, MAFG, NOV, } \\
\text { PDGFRB, PPP2CA }\end{array}$ \\
\hline
\end{tabular}


Table II. Continued.

Target genes and functions

\begin{tabular}{|c|c|c|c|}
\hline miRNA & Cell cycle & Apoptosis & Cell growth and proliferation \\
\hline hsa-miR-758-3p & $\begin{array}{l}\text { PARD6G, CCNG1, } \\
\text { AURKB, MPHOSPH1, } \\
\text { DLGAP5, DMTF1, BMP7, } \\
\text { NEK4, TACC1, TP53BP2, } \\
\text { VHL, WEE1, PPAPDC1B, } \\
\text { RBL1, PPP1CB, NUSAP1, } \\
\text { WWOX, ZAK, CDKN1B, } \\
\text { KHDRBS1, STAG2, } \\
\text { FOXN3, SGOL1, E2F6, } \\
\text { CLASP2, PDS5A, CD2AP, } \\
\text { CADM1, EID1, APPL1, } \\
\text { LIN9, STRN3, ING1, } \\
\text { LIG4, MCM3, MCM6 }\end{array}$ & $\begin{array}{l}\text { MALT1, UNC5D, CTSB, } \\
\text { RYBP, CADM1, APAF1, } \\
\text { PAK1, ZAK, KRT20, } \\
\text { APTX, IFT57, TNFRSF19, } \\
\text { BCL2L1, ATXN1, PERP, } \\
\text { UBE2Z, BNIP2, TIA1, } \\
\text { TIAL1, TP53BP2, } \\
\text { C10orf97, ACTN4, } \\
\text { RABEP1, EBAG9, BAG2, } \\
\text { BRE }\end{array}$ & $\begin{array}{l}\text { SLAMF1, STAT5B, KLF5, } \\
\text { BLZF1, CDC2L5, EBAG9, } \\
\text { SOCS6, NAMPT, } \\
\text { CDKN1B, CFDP1, } \\
\text { ENOX2, TCFL5, FGF7, } \\
\text { ID4, IGF1, ILK, LIFR, } \\
\text { NDN, CRIM1, POU3F2 }\end{array}$ \\
\hline
\end{tabular}

miRNAs, microRNAs; $\mathrm{H}_{2} \mathrm{O}_{2}$, hydrogen peroxide; HDPs, human dermal papilla cells.

Table III. Predicted target genes of the top five most downregulated miRNAs in $\mathrm{H}_{2} \mathrm{O}_{2}$-treated HDPs.

Target genes and functions

\begin{tabular}{|c|c|c|c|}
\hline \multirow[b]{2}{*}{ miRNA } & \\
\hline & Cell cycle & Apoptosis & Cell growth and proliferation \\
\hline hsa-miR-376c-3p & $\begin{array}{l}\text { JUB, CCNB3, CCNT2, } \\
\text { NOLC1, MTSS1, SIAH2, } \\
\text { AURKA, VHL, HMGA2, } \\
\text { CHAF1B, ERBB2IP, } \\
\text { PTEN, NPAT, PAFAH1B1, } \\
\text { GMNN, XRN1, } \\
\text { MPHOSPH8, SPIN1, } \\
\text { POLS, LZTS1, CHEK2, } \\
\text { SASS6, GADD45A, } \\
\text { MAPRE1, KANK1, } \\
\text { SASH1, APPL1, ANXA1, } \\
\text { HPGD, SEP14, MN1 }\end{array}$ & $\begin{array}{l}\text { EGLN3, RFFL, ACVR1C, } \\
\text { PARP4, CNTN4, } \\
\text { GADD45A, DAPK2, } \\
\text { TNFAIP8, SGMS1, ARF6, } \\
\text { MCL1, APIP, GULP1, } \\
\text { RHOT1, DUSP22, ROCK1, } \\
\text { ATXN1, SHB, SIAH2, } \\
\text { ACTC1, PHLDA2, } \\
\text { FASTKD3, CASP3, } \\
\text { CASP7, AIFM1, ATG12, } \\
\text { CD5L }\end{array}$ & $\begin{array}{l}\text { MARK4, CXCL5, BMPR2, } \\
\text { SSR1, STAT5A, TGFBR1, } \\
\text { TNFSF4, HMGA2, SOCS2, } \\
\text { SOCS6, CD86, CD47, } \\
\text { MORF4L2, DNAJA2, } \\
\text { CFDP1, TNFSF13B, } \\
\text { TBC1D8, SOCS4, FGF7, } \\
\text { FLT1, FLT3, RBM9, } \\
\text { ANXA1, IL2, CXCL10, } \\
\text { KRAS, NOV, IL17RB }\end{array}$ \\
\hline hsa-miR-423-5p & $\begin{array}{l}\text { JUB, CCNF, PKMYT1, } \\
\text { MPHOSPH1, DMTF1, } \\
\text { SUV39H1, TACC1, } \\
\text { DBF4B, BIN3, MAPK3, } \\
\text { MAPK4, PTCH1, RAP1A, } \\
\text { UPF1, TSPYL2, NF2, } \\
\text { PAFAH1B1, CINP, SUFU, } \\
\text { PFDN1, RBM5, CDKN1A, } \\
\text { HMG20B, TXNIP, SPIN1, } \\
\text { RCC1, FOXN3, LZTS1, } \\
\text { PMF1, E2F7, KCTD11, } \\
\text { SNF1LK, DAB2IP, CYLD, } \\
\text { DDX11, E2F2, EP300, }\end{array}$ & $\begin{array}{l}\text { BCL2L11, IL24, DIDO1, } \\
\text { RFFL, CLU, ACVR1C, } \\
\text { DAP, DEDD2, DOCK1, } \\
\text { E2F2, EIF5A, EP300, } \\
\text { ERN1, PTK2B, FOSL2, } \\
\text { CYFIP2, CIDEB, HIPK2, } \\
\text { SAP30BP, CARD10, HTT, } \\
\text { KCNIP3, HIP1, BIRC5, } \\
\text { IAPP, IL17A, LTBR, } \\
\text { MDM4, MLL, NGFR, } \\
\text { NDUFA13, PDCD1, } \\
\text { RNF216, PPARD, PRKCG, } \\
\text { BAG1, BCL2L1, RTKN, }\end{array}$ & $\begin{array}{l}\text { C19orf10, RAC2, SHC1, } \\
\text { STAT5A, STAT5B, } \\
\text { TGFBR2, VEGFB, } \\
\text { PROK1, CUL3, CDC2L5, } \\
\text { FGF18, NRP1, SOCS3, } \\
\text { SCGB3A1, S1PR2, } \\
\text { TAOK2, CIAO1, HTRA3, } \\
\text { NTN1, CLU, ADM, CSF1, } \\
\text { ADRA1D, KCTD11, CTF1, } \\
\text { ADRA2A, DDX11, DHPS, } \\
\text { S1PR3, PTK2B, FOXO1, } \\
\text { FLT3LG, RBM9, HOXC10, } \\
\text { VSX2, IGF1, IGFBP6, }\end{array}$ \\
\hline
\end{tabular}


Table III. Continued.

Target genes and functions

\begin{tabular}{|c|c|c|c|}
\hline miRNA & Cell cycle & Apoptosis & $\begin{array}{l}\text { Cell growth and } \\
\text { proliferation }\end{array}$ \\
\hline hsa-miR-423-5p & $\begin{array}{l}\text { PDS5A, NSL1, SGSM3, } \\
\text { BIRC5, INCENP, IRF1, } \\
\text { MCM7, MN1, NBL1, } \\
\text { NBN, SEP3, SEP8 }\end{array}$ & $\begin{array}{l}\text { NOD2, BNIP1, BOK, } \\
\text { PRDX2, CACNA1A, } \\
\text { BCL2L14, BIRC7, CASP2, } \\
\text { PLA2G6, TRAF7, AIFM2, } \\
\text { LGALS12, RIPK1, } \\
\text { TNFSF12, TNFRSF14, } \\
\text { TNFRSF10D, SQSTM1, } \\
\text { NOL3, BMF, DNAJA3, } \\
\text { TAOK2, NTN1, BRE }\end{array}$ & $\begin{array}{l}\text { KRT6A, MAFG, ODC1, } \\
\text { PDGFRB, C9orf127, } \\
\text { IL17RB, CCDC88A, } \\
\text { PRKCQ }\end{array}$ \\
\hline hsa-miR-3665 & - & - & - \\
\hline hsa-miR-20a-5p & $\begin{array}{l}\text { PARD6B, JUB, RUNX3, } \\
\text { CDC23, PCAF, BCL10, } \\
\text { CCND2, CCNG2, RNF8, } \\
\text { CCNT2, PKMYT1, } \\
\text { ZNF830, NEK9, KIF23, } \\
\text { RASSF2, RB1CC1, SEP7, } \\
\text { CDC25A, DLEC1, } \\
\text { DMTF1, PAPD5, BMP2, } \\
\text { STK11, TACC1, BUB1, } \\
\text { VHL, WEE1, PTP4A1, } \\
\text { SUV39H2, MAP9, PPP6C, } \\
\text { ERBB2IP, MAPK1, } \\
\text { MAPK4, PCNP, PTEN, } \\
\text { RAD17, RAP1A, RB1, } \\
\text { RBL1, RBL2, CCND1, } \\
\text { TIPIN, SEP6, NPAT, } \\
\text { PAFAH1B1, FZR1, ZAK, } \\
\text { XRN1, CHAF1A, } \\
\text { CDKN1A, TXNIP, CETN2, } \\
\text { SEP2, CIT, TUSC2, } \\
\text { SNF1LK, CYLD, E2F1, } \\
\text { E2F3, FANCD2, C11orf82, } \\
\text { HEPACAM, MAPRE1, } \\
\text { MAPRE3, SEP9, PDS5A, } \\
\text { SASH1, CLASP1, } \\
\text { RABGAP1, GAK, NSL1, } \\
\text { APPL1, FBXO5, CKAP2, } \\
\text { PDCD4, C13orf15, GRLF1, } \\
\text { RACGAP1, BIRC5, } \\
\text { TMPRSS11A, IRF1, MCC, } \\
\text { MCM3, NBL1 }\end{array}$ & $\begin{array}{l}\text { NOD1, FASTK, EGLN3, } \\
\text { NLRP3, E2F1, EIF4G2, } \\
\text { C11orf82, CARD8, ACIN1, } \\
\text { FAIM2, SULF1, RYBP, } \\
\text { SGMS1, MAGI3, CKAP2, } \\
\text { GJA1, TNFRSF21, } \\
\text { PDCD4, BIRC5, APP, } \\
\text { MCL1, MDM4, MAP3K5, } \\
\text { BCL2L15, OSM, } \\
\text { SH3GLB1, PDE1B, ZAK, } \\
\text { RHOT1, BCAP29, } \\
\text { PTGER3, BCL2, PERP, } \\
\text { ISG20L1, BNIP2, } \\
\text { TNFAIP3, SLTM, } \\
\text { C10orf97, RNF34, FXR1, } \\
\text { CASP2, PLA2G6, CASP7, } \\
\text { CASP8, CUL1, AIFM2, } \\
\text { TNFRSF10D, } \\
\text { TNFRSF10B, IER3, } \\
\text { SQSTM1, SGPL1, } \\
\text { TAX1BP1, BCL10, } \\
\text { RABEP1, ATG12, DEDD, } \\
\text { CD5L, STK17B, } \\
\text { TP53INP1, ATG5, LITAF, } \\
\text { TRAF4, SLK }\end{array}$ & $\begin{array}{l}\text { PURA, BCL2, CXCL5, } \\
\text { BMPR2, SSR1, TBX3, } \\
\text { TGFBR2, TSG101, } \\
\text { CAMK2D, ITCH, CDCA7, } \\
\text { BRMS1L, CUL3, CCND2, } \\
\text { S1PR2, SOCS6, } \\
\text { ARHGEF11, DNAJA2, } \\
\text { CFDP1, HOXB13, } \\
\text { MORF4L1, TBC1D8, } \\
\text { CHRNA7, S1PR3, ERBB3, } \\
\text { HEPACAM, FGF4, FGF7, } \\
\text { LRP12, IGFBP7, LIF, } \\
\text { LIFR, OSM, DERL2, } \\
\text { CRIM1, ING3, TIPIN, } \\
\text { PPP2CA }\end{array}$ \\
\hline hsa-miR-29c-3p & $\begin{array}{l}\text { PPM1D, CDC23, CCND2, } \\
\text { CCNF, UBA3, CCNT2, } \\
\text { AURKB, HDAC4, } \\
\text { DCLRE1A, SUPT5H, } \\
\text { TACC1, PTP4A1, CDC7, } \\
\text { SMPD3, RCC2, PTEN, } \\
\text { NEDD9, ZAK, XRN1, }\end{array}$ & $\begin{array}{l}\text { FEM1B, HTATIP2, IL24, } \\
\text { SLC5A8, AHR, FOXO3, } \\
\text { PPP1R13B, SIRT1, } \\
\text { ZNF346, CECR2, BIRC2, } \\
\text { FAS, IL2RA, MCL1, } \\
\text { SH3GLB1, ZAK, CYCS, } \\
\text { DIABLO, DUSP22, }\end{array}$ & $\begin{array}{l}\text { BIRC6, PURA, TRAF5, } \\
\text { VEGFA, EPC1, CDC7, } \\
\text { CREG1, CCND2, } \\
\text { MORF4L2, CDK2, } \\
\text { MORF4L1, CHRNA7, } \\
\text { RBM9, IFNG, IGF1, LIF, } \\
\text { LIFR, NDN, NEDD9, }\end{array}$ \\
\hline
\end{tabular}


Table III. Continued.

Target genes and functions

\begin{tabular}{|c|c|c|c|}
\hline \multirow[b]{2}{*}{ miRNA } & \\
\hline & Cell cycle & Apoptosis & Cell growth and proliferation \\
\hline \multirow[t]{8}{*}{ hsa-miR-29c-3p } & CDK2, CDK6, HTATIP2, & BIRC6, BAK1, ATXN1, & PDGFRB, ING3, PPP2CA \\
\hline & GREF1, STAG2, SEP9, & CIDEC, SGK1, ISG20L1, & \\
\hline & DBF4, MAPRE2, FOXN3, & TNFAIP3, TNFRSF1A, & \\
\hline & E2F7, SLC5A8, AHR, & TRAF5, FAM130A1, & \\
\hline & VASH1, MAPRE1, & CASP7, AIFM2, BMF, & \\
\hline & CLASP2, SEP6, & RABEP1, TP53INP1, & \\
\hline & PPP1R13B, MCC, MLF1, & TRAF4, SLK & \\
\hline & NASP & & \\
\hline
\end{tabular}

miRNAs, microRNAs; $\mathrm{H}_{2} \mathrm{O}_{2}$, hydrogen peroxide; HDPs, human dermal papilla cells.

programmed cell death (GO:0012501), regulation of apoptotic process (GO:0042981), regulation of cell death (GO:0010941), regulation of execution phase of apoptosis (GO:1900117) and regulation of programmed cell death (GO:0043067). Cell growth-related and cell proliferation-related GO included positive regulation of cell proliferation (GO:0008284), regulation of cell growth (GO:0001558), regulation of cell proliferation (GO:0042127) and regulation of growth (GO:0040008).

\section{Discussion}

ROS, such as $\mathrm{H}_{2} \mathrm{O}_{2}$, are generated as reactive byproducts of cellular metabolism in the mitochondria. The intracellular level of ROS is distinctly regulated by the cellular antioxidant system, including non-enzymatic and enzymatic antioxidants (27). The ROS level is increased in response to environmental stresses, including UV irradiation, toxic chemicals, heat and even high glucose concentrations $(27,28)$. High levels of ROS induce cell cycle arrest, senescence and apoptosis due to ROS damage of cellular membranes, lipids, proteins and DNA (7-10). In the present study, and in others previously, it has been identified that $\mathrm{H}_{2} \mathrm{O}_{2}$ induces growth arrest in HDPs (Fig. 1) (29-31). The $\mathrm{H}_{2} \mathrm{O}_{2}$-induced growth arrest occurs later within the cascade of events activated in response to $\mathrm{H}_{2} \mathrm{O}_{2}$ treatment. Previous investigations identified $\mathrm{H}_{2} \mathrm{O}_{2}$-responsive miRNAs, including miR-34 and miR-145, which also are implicated in the ROS-responsive pathway $(32,33)$. Therefore, miRNA appears to be required for $\mathrm{H}_{2} \mathrm{O}_{2}$-dependent growth arrest.

The present study identified 68 miRNAs that were regulated by $\mathrm{H}_{2} \mathrm{O}_{2}$ in HDPs (Fig. 2). miR-193-3p and miR-29b increased in $\mathrm{H}_{2} \mathrm{O}_{2}$-treated HDPs and induced apoptosis by targeting MCL-1 $(34,35)$. MCL-1 is a $B C L-2$ family member that is involved in mitochondria-dependent intrinsic apoptosis (36). MCL-1 represses apoptosis by preventing the formation of mitochondrial membrane potential (36). Our data, together with those of Lin et al (15), demonstrated that miR-193a-3p increased in response to ROS and subsequently induced cell death. A previous study revealed that miR-30a-5p was upregulated by $\mathrm{H}_{2} \mathrm{O}_{2}$, it repressed autophagy by targeting beclin-1 and eventually induced apoptosis (37). In the present study, it was demonstrated that miR-20a-5p and miR-423-5p were repressed by $\mathrm{H}_{2} \mathrm{O}_{2}$ in HDPs (Table I). miR-20a-5p had multiple target genes, including $B N I P 2, A P P, A S K 1$ and $T N K S 2$. Therefore, miR-20a-5p regulated proliferation, migration, invasion and inflammation by the regulation of its target genes (38-41). miR-424-5p targeted p21Cip1/Waf1, which functions in proliferation and G1 phase transition (42).

Numerous studies have demonstrated that ROS, including $\mathrm{H}_{2} \mathrm{O}_{2}$, induces intrinsic apoptosis (43-45). $\mathrm{H}_{2} \mathrm{O}_{2}$-induced apoptosis is regulated by mitochondrial membrane permeability, which is regulated by the $B C L-2$ family (36). The anti-apoptotic $B C L-2$ family, including BCL2L10,BCL2L11, BCL2L2 and $B C L 10$ were predicted as targets of miRNAs that were upregulated by $\mathrm{H}_{2} \mathrm{O}_{2}$ (Table II). The pre-apoptotic $B C L-2$ family members $B O K$ and $B A K 1$ were predicted as targets of miRNAs that were downregulated by $\mathrm{H}_{2} \mathrm{O}_{2}$ (Table III). Cell cycle regulating proteins, such as cyclins and $\mathrm{CDK}$, function during each phase of the cell cycle (G1, S, G2 and M) (46). CCNA1 and CDK2 are required for the regulation of the $\mathrm{G} 2 / \mathrm{M}$ phase $(47,48)$. The results of the present study predict that hsa-miR-30a-5p and hsa-miR-29b-3p target $C C N A 1$ and $C D K 2$. These data suggest that $\mathrm{H}_{2} \mathrm{O}_{2}$-mediated growth arrest and cell death in HDPs is associated with the changes in expression of specific miRNAs.

Bioinformatics analysis of miRNA expression profiles, miRNA target genes and the GO of target genes provided a more holistic view of the underlying cellular mechanisms that occur in response to $\mathrm{H}_{2} \mathrm{O}_{2}$-induced growth arrest and apoptosis. The identification of miRNAs and their putative targets may offer new therapeutic strategies for $\mathrm{H}_{2} \mathrm{O}_{2}$-induced hair follicle disorders, such as hair loss.

\section{Acknowledgements}

The authors are grateful to all the members of our research group for their support and advice regarding this study. This study was supported by the KU Research Professor Program of Konkuk University.

\section{References}

1. Oliver RF and Jahoda CA: Dermal-epidermal interactions. Clin Dermatol 6: 74-82, 1988. 
2. Driskell RR, Clavel C, Rendl M and Watt FM: Hair follicle dermal papilla cells at a glance. J Cell Sci 124: 1179-1182, 2011.

3. Greco V, Chen T, Rendl M, Schober M, Pasolli HA, Stokes N, Dela Cruz-Racelis J and Fuchs E: A two-step mechanism for stem cell activation during hair regeneration. Cell Stem Cell 4: 155-169, 2009

4. Oshimori $\mathrm{N}$ and Fuchs E: Paracrine TGF- $\beta$ signaling counterbalances BMP-mediated repression in hair follicle stem cell activation. Cell Stem Cell 10: 63-75, 2012.

5. Enshell-Seijffers D, Lindon C, Kashiwagi M and Morgan BA: $\beta$-Catenin activity in the dermal papilla regulates morphogenesis and regeneration of hair. Dev Cell 18: 633-642, 2010.

6. Enshell-Seijffers D, Lindon C, Wu E, Taketo MM and Morgan BA: $\beta$-Catenin activity in the dermal papilla of the hair follicle regulates pigment-type switching. Proc Natl Acad Sci USA 107: 21564-21569, 2010.

7. Hamanaka RB, Glasauer A, Hoover P, Yang S, Blatt H, Mullen AR, Getsios S, Gottardi CJ, DeBerardinis RJ, Lavker RM and Chandel NS: Mitochondrial reactive oxygen species promote epidermal differentiation and hair follicle development. Sci Signal 6: ra8, 2013.

8. Wood JM, Decker H, Hartmann H, Chavan B, Rokos H, Spencer JD, Hasse S, Thornton MJ, Shalbaf M, Paus R and Schallreuter KU: Senile hair graying: $\mathrm{H}_{2} \mathrm{O}_{2}$-mediated oxidative stress affects human hair color by blunting methionine sulfoxide repair. FASEB J 23: 2065-2075, 2009.

9. Trüeb RM: Oxidative stress in ageing of hair. Int J Trichology 1: 6-14, 2009.

10. Luanpitpong S, Nimmannit U, Chanvorachote P, Leonard SS Pongrakhananon V, Wang L and Rojanasakul Y: Hydroxyl radical mediates cisplatin-induced apoptosis in human hair follicle dermal papilla cells and keratinocytes through Bcl-2-dependent mechanism. Apoptosis 16: 769-782, 2011.

11. Chekulayeva LV, Shevchuk IN, Chekulayev VA and Ilmarinen K: Hydrogen peroxide, superoxide, and hydroxyl radicals are involved in the phototoxic action of hematoporphyrin derivative against tumor cells. J Environ Pathol Toxicol Oncol 25: 51-77, 2006.

12. Koruk M, Taysi S, Savas MC, Yilmaz O, Akcay F and Karakok M: Oxidative stress and enzymatic antioxidant status in patients with nonalcoholic steatohepatitis. Ann Clin Lab Sci 34: 57-62, 2004.

13. Bartosz G: Non-enzymatic antioxidant capacity assays: limitations of use in biomedicine. Free Radic Res 44: 711-720, 2010.

14. Lukiw WJ and Pogue AI: Induction of specific micro RNA (miRNA) species by ROS-generating metal sulfates in primary human brain cells. J Inorg Biochem 101: 1265-1269, 2007.

15. Lin Y, Liu X, Cheng Y, Yang J, Huo Y and Zhang C: Involvement of MicroRNAs in hydrogen peroxide-mediated gene regulation and cellular injury response in vascular smooth muscle cells J Biol Chem 284: 7903-7913, 2009.

16. Wang Z, Liu Y, Han N, Chen X, Yu W, Zhang W and Zou F: Profiles of oxidative stress-related microRNA and mRNA expression in auditory cells. Brain Res 1346: 14-25, 2010.

17. He L and Hannon GJ: MicroRNAs: small RNAs with a big role in gene regulation. Nat Rev Genet 5: 522-531, 2004.

18. Winter J and Diederichs S: MicroRNA biogenesis and cancer. Methods Mol Biol 676: 3-22, 2011

19. Papagiannakopoulos T and Kosik KS: MicroRNAs: regulators of oncogenesis and stemness. BMC Med 6: 15, 2008.

20. Lin SL, Chiang A, Chang D and Ying SY: Loss of mir-146a function in hormone-refractory prostate cancer. RNA 14 : 417-424, 2008

21. Kogo R, Mimori K, Tanaka F, Komune S and Mori M: Clinical significance of miR-146a in gastric cancer cases. Clin Cancer Res 17: 4277-4284, 2011.

22. Hou Z, Xie L, Yu L, Qian X and Liu B: MicroRNA-146a is down-regulated in gastric cancer and regulates cell proliferation and apoptosis. Med Oncol 29: 886-892, 2012.

23. Teta M, Choi YS, Okegbe T, Wong G, Tam OH, Chong MM, Seykora JT, Nagy A, Littman DR, Andl T and Millar SE: Inducible deletion of epidermal Dicer and Drosha reveals multiple functions for miRNAs in postnatal skin. Development 139: 1405-1416, 2012.

24. Mardaryev AN, Ahmed MI, Vlahov NV, Fessing MY, Gill JH, Sharov AA and Botchkareva NV: Micro-RNA-31 controls hair cycle-associated changes in gene expression programs of the skin andhair follicle. FASEB J 24: 3869-3881, 2010.

25. Goodarzi HR, Abbasi A, Saffari M, Fazelzadeh Haghighi M, Tabei MB and Noori Daloii MR: Differential expression analysis of balding and nonbalding dermal papilla microRNAs in male pattern baldness with a microRNA amplification profiling method. Br J Dermatol 166: 1010-1016, 2012.
26. Lee JP, Cha HJ, Lee KS, Lee KK, Son JH, Kim KN, Lee DK and An S: Phytosphingosine-1-phosphate represses the hydrogen peroxide-induced activation of c-Jun N-terminal kinase in human dermal fibroblasts through the phosphatidylinositol 3-kinase/Akt pathway. Arch Dermatol Res 304: 673-678, 2012.

27. Cheng Z and Ristow M: Mitochondria and metabolic homeostasis. Antioxid Redox Signal 19: 240-242, 2013.

28. Caputo F, Vegliante R and Ghibelli L: Redox modulation of the DNA damage response. Biochem Pharmacol 84: 1292-1306, 2012.

29. Li M, Zhao L, Liu J, Liu AL, Zeng WS, Luo SQ and Bai XC: Hydrogen peroxide induces G2 cell cycle arrest and inhibits cell proliferation in osteoblasts. Anat Rec (Hoboken) 292: 1107-1113, 2009.

30. Cho SD, Li G, Hu H, Jiang C, Kang KS, Lee YS, Kim SH and Lu J: Involvement of c-Jun N-terminal kinase in G2/M arrest and caspase-mediated apoptosis induced by sulforaphane in DU145 prostate cancer cells. Nutr Cancer 52: 213-224, 2005.

31. He L, Nan MH, Oh HC, Kim YH, Jang JH, Erikson RL, Ahn JS and Kim BY: Asperlin induces $\mathrm{G}_{2} / \mathrm{M}$ arrest through ROS generation and ATM pathway in human cervical carcinoma cells. Biochem Biophys Res Commun 409: 489-493, 2011.

32. Iekushi K, Seeger F, Assmus B, Zeiher AM and Dimmeler S: Regulation of cardiac microRNAs by bone marrow mononuclear cell therapy in myocardial infarction. Circulation 125: 1765-1773, S1-S7, 2012.

33. Li R, Yan G, Li Q, Sun H, Hu Y, Sun J and Xu B: MicroRNA-145 protects cardiomyocytes against hydrogen peroxide $\left(\mathrm{H}_{2} \mathrm{O}_{2}\right)$-induced apoptosis through targeting the mitochondria apoptotic pathway. PLoS One 7: e44907, 2012

34. Chen J, Zhang X, Lentz C, Abi-Daoud M, Paré GC, Yang X, Feilotter HE and Tron VA: miR-193b regulates Mcl-1 in melanoma. Am J Pathol 179: 2162-2168, 2011.

35. Mott JL, Kobayashi S, Bronk SF and Gores GJ: mir-29 regulates Mcl-1 protein expression and apoptosis. Oncogene 26: 6133-6140, 2007.

36. Yang-Yen HF: Mcl-1: a highly regulated cell death and survival controller. J Biomed Sci 13: 201-204, 2006.

37. Zhu H, Wu H, Liu X, Li B, Chen Y, Ren X, Liu CG and Yang JM: Regulation of autophagy by a beclin 1-targeted microRNA, miR-30a, in cancer cells. Autophagy 5: 816-823, 2009

38. Fan X, Liu Y, Jiang J, Ma Z, Wu H, Liu T, Liu M, Li X and Tang $\mathrm{H}$ : miR-20a promotes proliferation and invasion by targeting APP in human ovarian cancer cells. Acta Biochim Biophys Sin (Shanghai) 42: 318-324, 2010.

39. Chai H, Liu M, Tian R, Li X and Tang H: miR-20a targets BNIP2 and contributes chemotherapeutic resistance in colorectal adenocarcinoma SW480 and SW620 cell lines. Acta Biochim Biophys Sin (Shanghai) 43: 217-225, 2011

40. Philippe L, Alsaleh G, Pichot A, Ostermann E, Zuber G, Frisch B, Sibilia J, Pfeffer S, Bahram S, Wachsmann D and Georgel P: MiR-20a regulates ASK1 expression and TLR4-dependent cytokine release in rheumatoid fibroblast-like synoviocytes. Ann Rheum Dis 72: 1071-1079, 2013.

41. Kang HW, Wang F, Wei Q, Zhao YF, Liu M, Li X and Tang H: miR-20a promotes migration and invasion by regulating TNKS2 in human cervical cancer cells. FEBS Lett 586: 897-904, 2012.

42. Lin J, Huang S, Wu S, Ding J, Zhao Y, Liang L, Tian Q, Zha R, Zhan R and He X: MicroRNA-423 promotes cell growth and regulates $\mathrm{G}(1) / \mathrm{S}$ transition by targeting $\mathrm{p} 21 \mathrm{Cip} 1 / \mathrm{Waf} 1$ in hepatocellular carcinoma. Carcinogenesis 32: 1641-1647, 2011.

43. Lin HJ, Wang X, Shaffer KM, Sasaki CY and Ma W: Characterization of $\mathrm{H}_{2} \mathrm{O}_{2}$-induced acute apoptosis in cultured neural stem/progenitor cells. FEBS Lett 570: 102-106, 2004.

44. Herrera B, Alvarez AM, Sánchez A, Fernández M, Roncero C, Benito M and Fabregat I: Reactive oxygen species (ROS) mediates the mitochondrial-dependent apoptosis induced by transforming growth factor (beta) in fetal hepatocytes. FASEB J 15: 741-751, 2001.

45. Cai J and Jones DP: Superoxide in apoptosis. Mitochondrial generation triggered by cytochrome c loss. J Biol Chem 273: 11401-11404, 1998.

46. Arellano M and Moreno S: Regulation of CDK/cyclin complexes during the cell cycle. Int J Biochem Cell Biol 29: 559-573, 1997.

47. Rivera A, Mavila A, Bayless KJ, Davis GE and Maxwell SA Cyclin A1 is a p53-induced gene that mediates apoptosis, G2/M arrest, and mitotic catastrophe in renal, ovarian, and lung carcinoma cells. Cell Mol Life Sci 63: 1425-1439, 2006.

48. Chung JH and Bunz F: Cdk2 is required for $\mathrm{p} 53$-independent G2/M checkpoint control. PLoS Genet 6: e1000863, 2010. 